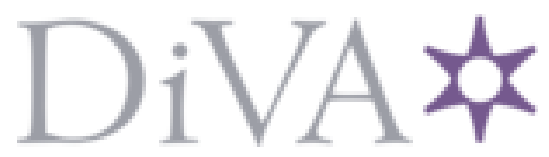

http://www.diva-portal.org

\title{
Postprint
}

This is the accepted version of a paper presented at 7th European Conference on Mobile Robots 2015, Lincoln, United Kingdom, 2-4 September, 2015.

Citation for the original published paper:

Gholami Shahbandi, S., Åstrand, B., Philippsen, R. (2015)

Semi-Supervised Semantic Labeling of Adaptive Cell Decomposition Maps in Well-Structured Environments.

In: 2015 European Conference on Mobile Robots (ECMR) Piscataway, NJ: IEEE Press http://dx.doi.org/10.1109/ECMR.2015.7324207

N.B. When citing this work, cite the original published paper.

Permanent link to this version:

http://urn.kb.se/resolve?urn=urn:nbn:se:hh:diva-29343 


\section{Semi-Supervised Semantic Labeling of Adaptive Cell Decomposition Maps in Well-Structured Environments}

\author{
Saeed Gholami Shahbandi \\ saesha@hh.se \\ Halmstad University, Sweden
}

\author{
Björn Åstrand \\ bjorn.astrand@hh.se \\ Halmstad University, Sweden
}

\author{
Roland Philippsen \\ roland.philippsen@hh.se \\ Halmstad University, Sweden
}

\begin{abstract}
We present a semi-supervised approach for semantic mapping, by introducing human knowledge after unsupervised place categorization has been combined with an adaptive cell decomposition of an occupancy map. Place categorization is based on clustering features extracted from raycasting in the occupancy map. The cell decomposition is provided by work we published previously, which is effective for the maps that could be abstracted by straight lines. Compared to related methods, our approach obviates the need for a low-level link between human knowledge and the perception and mapping sub-system, or the onerous preparation of training data for supervised learning. Application scenarios include intelligent warehouse robots which need a heightened awareness in order to operate with a higher degree of autonomy and flexibility, and integrate more fully with inventory management systems. The approach is shown to be robust and flexible with respect to different types of environments and sensor setups.
\end{abstract}

\section{INTRODUCTION}

Semantic labeling has been the nucleus of much robotics research in different application areas. The objective behind semantics is to elevate the robot's understanding by linking its world model to human related meanings. Semantics vary by context, such as human-robot interaction [1], [2] and object semantics for manipulation [3]. Arguably, the term "semantic map" is most commonly associated with semantic labeling of places in mobile robotics. A robot's understanding solely from the sensor perspective is limited to the level of appearance-based distinctions (e.g. from vision, laser, haptics). On the other hand, human knowledge often derives semantics from the functionality of the subject (objects or places). In general, a robot cannot acquire semantics unless there is a link between human knowledge and a robot's understanding. That is to say, human semantics must be introduced to robots. More intelligent behavior is enabled by exploiting the links with human knowledge embedded in a robot's semantic model.

Automatic Inventory and Mapping of Stock (AIMS) is a project that targets the domain of intelligent warehouses, by leveraging a synergic application of logistics management and intelligent vehicles. In AIMS, a set of tools is developed for setting up and operating Automatic Guided Vehicles (AGV) in warehouses. The project covers a variety

This work was supported by the Swedish Knowledge Foundation and industry partners Kollmorgen, Optronic, and Toyota Material Handling Europe. It was carried out at the Center for Applied Intelligent Systems Research at Halmstad University, Sweden.

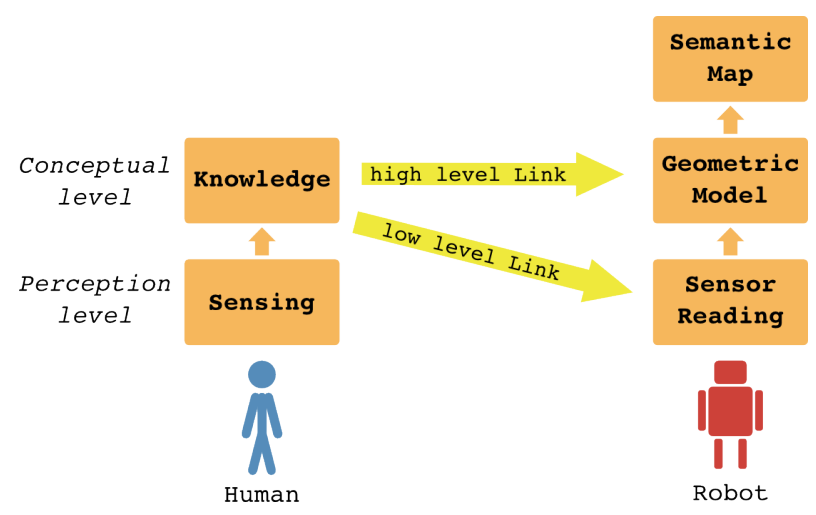

Fig. 1: Levels of semantic linkage between human knowledge and robot world model. Our method lifts the input required from humans to a higher level of abstraction by combining unsupervised place categorization with automatic spatial abstraction.

of tasks, from layout surveying to on-line inventory tracking. A central element for fulfilling the project objectives is the establishment of a robust and flexible semantic mapping framework. Our previous work [4], [5] focuses on spatial modelling methods well suited for semantic annotation and inference. In this present paper, we address the problem of providing human knowledge to robots for the purpose of semantic mapping.

Related Work: There is a significant body of work on semantic analysis of the kinds of environments relevant for AIMS. We discuss only the ones most pertinent to this paper, emphasizing the abstraction level where semantic links are introduced (Fig. 1).

Low level delivery of human defined semantic to robots covers research that provides training data with semantic labels. Examples of such work includes supervised semantic labeling of cell in an occupancy map [6], using image features and similarly range features for place labeling [7], through generation of a visual vocabulary based on features and employing Latent Dirichlet Allocation (LDA) [8], or learning of feature hierarchies at different abstraction levels [9].

On the other hand, some works provide semantic notation to the robot when it already has a map of the environment (high level link in Fig. 1). In such a case, either place seg- 
mentation and semantic annotation is performed manually (as it is provided on the location), or place detection and labeling is performed with a template matching approach. Examples of manual selection and annotation are; [10] explores an occupancy grid map through the Voronoi graph, [11] uses natural language based annotation on location, [12] segments and annotates of decomposed occupancy map, [13] annotates place with semantic label on the location using a laser pointer. Examples of matching a template with the model are; [14] builds a geometric model of the world, and looks for templates (e.g. walls, floors, or objects), [15] proposes an inference model over a 3D correlating objects framework with semantic labels, [16] provides a topological graph of the environment based on the concept of virtual door, [17] embeds semantics in kernels and the map is refined and segmented accordingly, [5] presents semantic labels as graph templates and matches to the connectivity map of the open space, [3] performs a segmentation and semantic labeling of objects in 3D data. The last example is the probabilistic framework for semantic inference proposed by [18] which covers a large variety of techniques on different levels.

The provided examples deliver reliable performance in specific application areas such as office environments. Application area of the AIMS project requires semantic labeling of the structure of the environment. Approaching semantic labeling through objects found in places seems less suitable. In addition to that, our solution is required to be generic, independent of training and robust to different configuration of environment and mapping. That explains why we are interested in postpoing the delivery of semantics to robot's model into higher level.

Our Approach: The focus of this work is the construction of a semantic map without providing semantic training data as an input to the system. The robot autonomously builds a high level spatial model of the world and instantiates it, without prior knowledge of the environment. This allows an abstraction of human semantics, instead of providing this knowledge through a training set. Human defined semantics are introduced at a high level, as annotations of the model's instances. These semantics are provided as labels accompanied with their functionality and possible inter relations between them. This allows the robot to perform an inference over its model and provided semantics, resulting in the desired semantic map. The contribution of this work is the autonomous generation of a high level representation that allows an efficient abstraction of the semantics based on human input.

\section{MethoD}

The end goal of this work is the generation of a semantic map. For this purpose, an abstract spatial representation of the environment is provided and fused with an unsupervised place categorization of each cell of the occupancy map. While the robot generates and carries a high level spatialconceptual model of the environment, human semantics is introduced. The semantics notion is provided in a high level and abstract form. The semantics relates the place categories

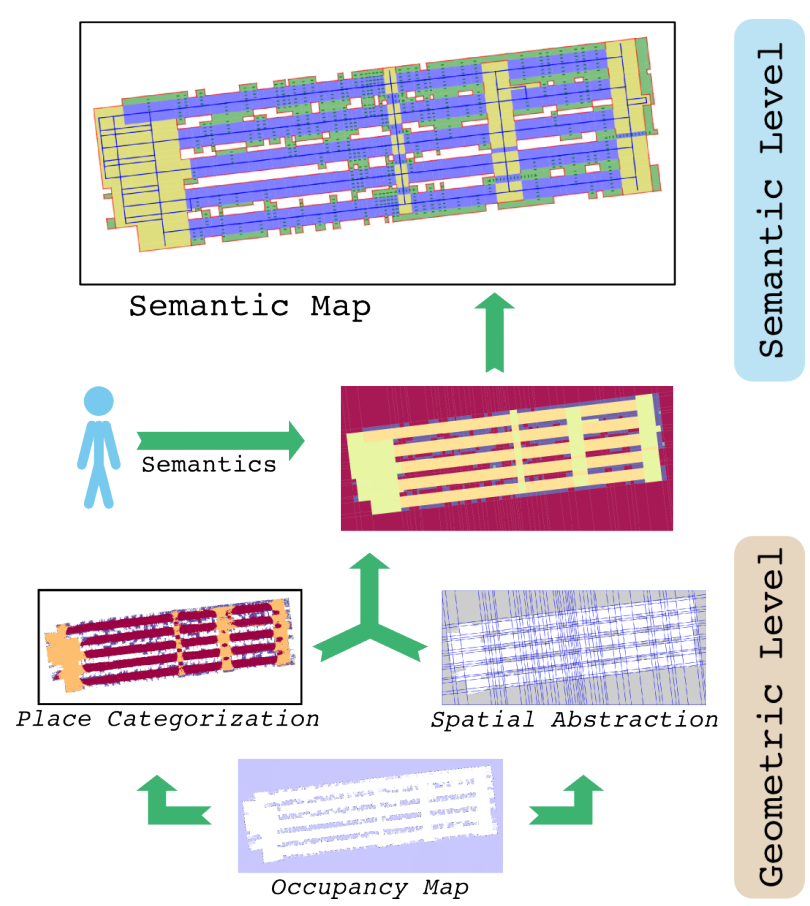

Fig. 2: Overall flow of the proposed method. The geometric level runs without human input and produces a spatially abstracted map annotated with place categories. The semantic level leverages human refinement of the geometric information to infers a higher-level semantic map.

in the robot's model with human-defined functionalities and attributes of the places. This connection enables an inference over the robot's spatial-conceptual model, into a semantic map. The developed semantic map maintains the abstract spatial model of the environment along with the semantic labels of each entity in the model, accompanied with inferred inter relation between entities and their functionalities.

This process is depicted in Fig. 2. For each map the two processes of spatial abstraction and place categorization is done independently. Spatially abstract representation of the map is achieved by an Adaptive Cell Decomposition represented by two data structures of subdivision and adjacency graph (see our previous work[4]). The other process performs an unsupervised place categorization of the map, by clustering places according to their geometric shape represented by a feature set proposed by [6]. This feature set is based on the assumption that places are identifiable based on their shape as reflected in the sensor reading. Furnishing the spatial model with place categories, the model holds instances ready to receive semantic annotations, designed according to the environment and in correspondence to the place categorization. With simple inference rules the robot is able to realize relations among neighboring instances of the model. The inference over the semantic labels in the context of the robot's model results in the desired semantic map.

\section{A. Unsupervised Place Categorization}

Mozos et al. proposed a method of supervised semantic labeling of places [6] based on geometric descriptors and 


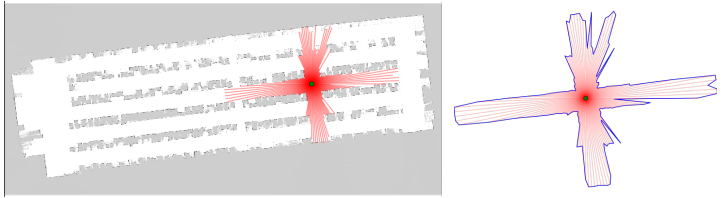

Fig. 3: Raycast example in a warehouse.

Mean and standard deviation of beams' length.
Mean and standard deviation of differences between consecutive
beams' length.
Area $(\mathrm{A})$ and perimeter $(\mathrm{P})$ of the surface covered by the raycast, and
the ratio $\left(P^{2} / A\right)$.
Number of gaps between consecutive beams, defined by a threshold.
Kurtosis, a measure of elongation of the covered area.
$P C 1$ and $P C 2$ of the PCAs from the raycast. The ratio $(P C 1 / P C 2)$
and the offset between origin of the PCA frame and raycasting point.

TABLE I: Thirteen features extracted from raycast proposed by [6].

bootstrapped classifiers. From each cell of the occupancy map, laser readings are simulated by raycasting (Fig. 3), and simple geometric features (table I) are extracted from the raycasts. In their method, the map is divided into two parts, one for training classifiers and one for evaluation. The performance of this feature set is evaluated in office-like environments.

Place categorization in our work is inspired by their feature set. However, instead of classifying map cells based on training from manually labelled data, our work relies on unsupervised clustering. By using this set of features we rely on the same assumption as [6], namely that places in the map are distinguishable based on their shape as perceived by the sensor. Our unsupervised approach liberates it from the need for specific and predefined semantic labels.

One of the advantages of using raycasts is that they sees far beyond the origin location. More importantly, there is no need for an assumption on the underlying structure of the environment for categorization of places (as opposed to training sets). Fig. 6a demonstrates the result of place categorization of an occupancy map from a real warehouse. In our current implementation, we use k-means clustering of the features presented in table $\mathrm{I}$, and the $k$ value is set according to type of the environment (i.e. more than the number of expected categories). However, note that our method does not rely on the specific clustering algorithm, and we expect to go beyond simple k-means in future work. Our current implementation assigns a single category label $\mathrm{L}\left(p_{i}\right)$ to each cell $p_{i}=\left(x_{i}, y_{i}\right)$ in the occupancy map. Future work may extend this to more expressive schemes, such as determining distributions over possible categories.

\section{B. Adaptive Cell Decomposition}

In previous work we developed an Adaptive Cell Decomposition of maps [4], a novel approach of abstraction and capturing the latent structure of the environment by adapting the decomposition to the spatial resolution of the structure. This structure could be generated by walls in an office environment, or the alignment of physical entities



Fig. 4: Detection of dominant orientations, using Continuous Wavelet Transform (CWT) of weighted Histogram of Oriented Gradients (HOG), modeled by Gaussian Mixture Model (GMM).

(crates containing articles) in a warehouse which typically lack walls where AGVs operate. This subsection summarizes the decomposition method and describes improvements and modifications introduced for this paper.

Dominant Orientations: Decomposition starts by finding the dominant orientations of the structure through the peaks in the weighted Histogram of Oriented Gradients (wHOG), given the number of expected orientations. We have removed the previous need to provide the number of dominant orientations by modelling the wHOG with a Gaussian Mixture Model (GMM). Dominant orientations are detected from the GMM modelled signal by a peak detection technique based on Continuous Wavelet Transform (CWT). Although CWT is known to be robust against noise by employing a range of wavelets with different scales, it is not sufficient for this case. This is due to cases where spikes appear close to strong peaks which correspond to dominant orientations. While it is desired to reject this kind of noise, there are similar stand-alone peaks that actually represent other dominant orientations, and which should not be filtered out. Therefore if CWT is used solely, the range of wavelet scales must be kept very broad. Here GMM acts as an aggressive Low Pass Filter, to merge noises into dominant orientations, while not completely removing important stand-alone peaks. That is to say, GMM simplifies and captures the outline of the histogram (See Fig. 4), resulting in a smoother signal. This allows wavelet scales to be more narrow, resulting in increased robustness. Dominant orientations are represented by the set $\Theta$

$$
\Theta:=\left\{\theta_{i} \mid i \in \mathbb{N}, \theta_{i} \in\left[-\frac{\pi}{2}, \frac{\pi}{2}\right]\right\}
$$

Cell Decomposition: A set of lines $\left(\mathcal{L}_{\text {ines }}\right)$ constructs a low level spatial representation of the environment's structure. This set underlies the cell decomposition.

$$
\mathcal{L}_{\text {ines }}:=\left\{y=m_{i} \times x+b_{i} \mid i \in \mathbb{N}, x, y, m_{i}, b_{i} \in \mathbb{R}\right\}
$$

As the gradient $m_{i}$ is already computed in the dominant orientations $\Theta$, the constant term $b_{i}$ (y-intercept) remains to be determined. This is achieved by radiography. Radiography is a projection of occupied cells of the map along the angles in $\Theta$. Peaks in the radiogram signals correspond to alignment of physical elements in the occupancy map along the angles in $\Theta$. For more details see [4]. 


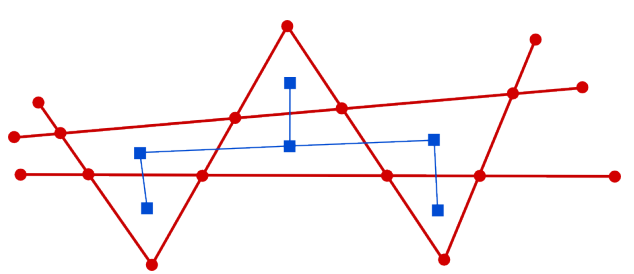

Fig. 5: Subdivision and adjacency graph. Thick red lines and red dots are edges and vertices of the subdivision. Thin blue lines and blue squares are links and nodes from adjacency graph.

Data Structures: Cell decomposition is generated over the line set $\mathcal{L}_{\text {ines }}$ and represents a higher level abstraction of the map. The cell decomposition is conveyed by two data structures, subdivision and adjacency graph (see Fig. 5).

The subdivision $\mathrm{S}$ is composed of 3 sets of $\mathcal{V}_{\text {ertices }}, \mathcal{E}_{\text {dges }}$ and $\mathcal{F}_{\text {aces }}$. A neighborhood function of edges $\mathrm{N}(e)$ and a membership function of faces $\mathrm{M}(f)$ are defined over those sets of subdivision.

$$
\begin{aligned}
& \mathrm{S}=\left(\mathcal{V}_{\text {ertices }}, \mathcal{E}_{\text {dges }}, \mathcal{F}_{\text {aces }}\right)=\left(\left\{v_{i}\right\},\left\{e_{i}\right\},\left\{f_{i}\right\}\right) \\
& v_{i}=\left(x_{i}, y_{i}\right), e_{i}=\left(v_{i l}, v_{i k}\right), f_{i}=\left\{e_{i j}\right\} \\
& \mathrm{N}\left(e_{i}\right)=\left\{\left(f_{i l}, f_{i k}\right) \mid\left(e_{i} \in f_{i l} \wedge e_{i} \in f_{i k}\right)\right\} \\
& \mathrm{M}\left(f_{i}\right)=\left\{\left(x_{i}, y_{i}\right) \mid\left(x_{i}, y_{i}\right) \text { inside } f_{i}\right\}
\end{aligned}
$$

The adjacency graph A has a neighborhood function of nodes $\mathrm{N}(n)$ operating on two sets of $\mathcal{N}_{\text {odes }}$ and $\mathcal{L}_{\text {inks }}$.

$$
\begin{aligned}
& \mathrm{A}=\left(\mathcal{N}_{\text {odes }}, \mathcal{L}_{\text {inks }}\right)=\left(\left\{n_{i}\right\},\left\{l_{i}\right\}\right) \\
& n_{i}=\left(x_{i}, y_{i}\right), l_{i}=\left(n_{i 1}, n_{i 2}\right) \\
& \mathrm{N}\left(n_{i}\right)=\left\{n_{j} \mid l_{k} \in \mathcal{L}_{\text {inks }}, l_{k}=\left(n_{i}, n_{j}\right)\right\}
\end{aligned}
$$

The two data structures, subdivision and adjacency graph, are dual in nature (see Fig. 5). That is to say, each face and edge in $\mathrm{S}$ are dual to a node and link in AG respectively. Duality is addressed by a set of dual functions $\mathrm{D}(\cdot)$.

$$
\mathrm{D}(n) \in\{f\}, \mathrm{D}(f) \in\{n\}, \mathrm{D}(l) \in\{e\}, \mathrm{D}(e) \in\{l\}
$$

An adaptive cell decomposition of a warehouse map is illustrated in Fig. $6 \mathrm{~b}$ only by its decomposing lines $\left(\mathcal{L}_{\text {ines }}\right)$.

Subdivision Annotation: Originally, in [4], the occupancy of the faces are referred to as their labels. Here, we rely on the unsupervised place categorization instead. In the current implementation, faces in the subdivision inherit their labels $\mathrm{L}(f)$ from underlying cells (pixels) of the occupancy map in a voting process (winner takes all). Future work may extend this to more elaborate schemes, for example to increase robustness and flexibility.

$$
\mathrm{L}\left(f_{i}\right)=\operatorname{Mode}\left\{L\left(p_{i}\right) \mid p_{i} \in \mathrm{M}\left(f_{i}\right)\right\}
$$

Inheritance of the labels from occupancy map to the subdivision is demonstrated in Fig. 6c.

\section{Introducing Human Semantics}

At this point the robot has a rich representation model of the environment. Yet this model requires semantics from human knowledge in order for the robot to benefit from its

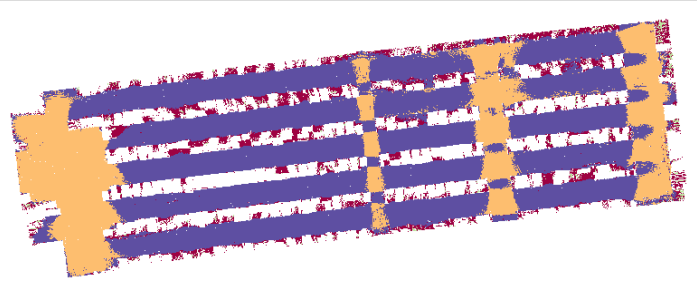

(a) place categorization

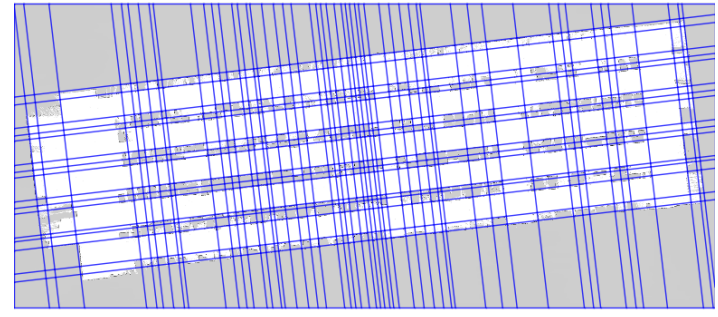

(b) adaptive cell decomposition

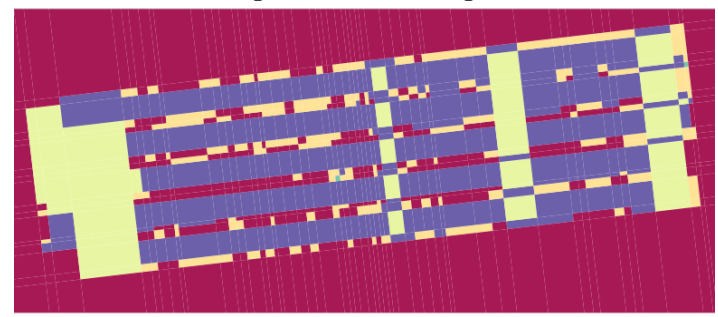

(c) subdivision annotation

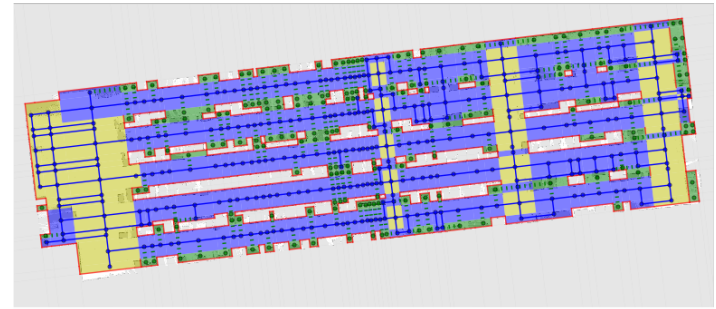

(d) semantic map

Fig. 6: 6b demonstrates the decomposition of an occupancy map, 6a shows unsupervised place categorization, $6 c$ demonstrates the annotation of the decomposition (6b) with categories from $6 \mathrm{a}$, followed by $6 \mathrm{~d}$ demonstrating one possible inference (table II) over the linked knowledge of human to robot's model. Note that only colors from $6 \mathrm{~d}$ have semantic meanings, and they correspond to those colors of figure $7 \mathrm{a}$.

model's full functionality. The result of this link heightens the robot's awareness of its environment.

An example of realizing this objective is to deliver to the robot a set of semantic labels, their functionalities and corresponding relations. We propose such labels for a warehouse in table II and visualized in Fig. 7. This is one way, and to our experience, the most straight forward way of labeling a warehouse environment. While table II is highly abstract, it is sufficiently descriptive to enable the robot inferring human related semantic labels from its model. For example type of relations between neighboring instances of the model could 


\begin{tabular}{|l|l|c|}
\hline Label & Functionality & Connect-ability \\
\hline co: corridor & drivable \& storage access & co \& ju \& pc \\
\hline ju: junctions & drivable \& connection & co \& ju \\
\hline pc: pallet cells & storage & co \\
\hline un: unidentified & - & None \\
\hline
\end{tabular}

TABLE II: Semantic labels, their attributes and functionality manually designed for warehouses.

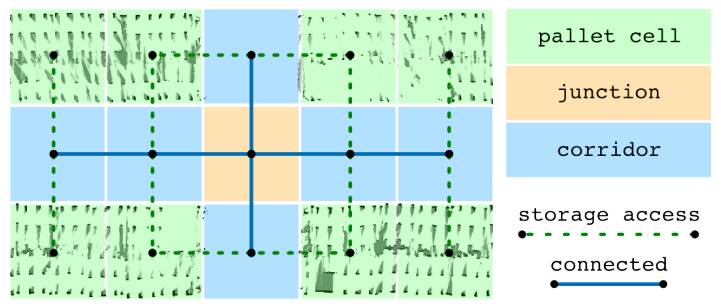

(a) semantic labels

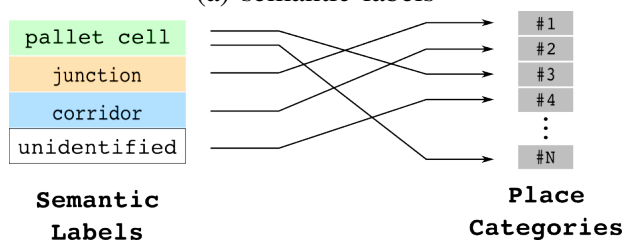

(b) assigning semantics to place categories

Fig. 7: In this figure, human semantics designated to places in warehouse are demonstrated according to table II. Types of connections between different places as annotated, could be inferred from the same table.

be inferred.

Edges and links in the subdivision and adjacency graph have attributes, indicating the type of connections between neighboring faces/nodes. The attributes depend on the labels of neighboring faces, and can be inferred from the "Connectability" column of table II. A cogent edge implies an obstruction between neighboring faces (and dual nodes), which means that the dual link has a disconnected attribute. An invalid edge implies an open access between neighboring faces (and dual nodes), and consequently the dual link is connected. The third type of edge/link attribute, storage access, indicates that a drivable face (corridor) is neighboring a storage face (pallet cell). One generic realization of inference over table II is given by the Cogency function of edges $\mathrm{C}(e)$ and connectivity function of links $\mathrm{C}(l)$.

$$
\begin{gathered}
\mathrm{C}\left(e_{i}\right)= \begin{cases}\text { cogent } & \text { if un } \in N\left(e_{i}\right) \\
\text { cogent } & \text { if } N\left(e_{i}\right)=\{\mathrm{ju} \wedge \mathrm{pc}\} \\
\text { storage access } & \text { if } N\left(e_{i}\right)=\{\mathrm{co} \wedge \mathrm{pc}\} \\
\text { invalid } & \text { otherwise }\end{cases} \\
\mathrm{C}\left(l_{i}\right)= \begin{cases}\text { disconnected } & \text { if } \mathrm{D}\left(\mathrm{l}_{\mathrm{i}}\right)=\text { cogent } \\
\text { storage access } & \text { if } \mathrm{D}\left(\mathrm{l}_{\mathrm{i}}\right)=\text { storage access } \\
\text { connected } & \text { if } \mathrm{D}\left(\mathrm{l}_{\mathrm{i}}\right)=\text { invalid }\end{cases}
\end{gathered}
$$

Fig. 7 visualizes the interpretation of the cogency and connectivity functions. The decomposition of faces and their place categories in Fig. 7 is already available in the



Fig. 8: Region segmentation using Gabor decomposition. The map represents a warehouse environment of size $\sim 190 \mathrm{~m} \times$ $270 m$.

robot's model of the environment. The link between human semantics (table II) and robot model allows the robot to perceive its surrounding similar to humans. At this stage of our work, the links between human semantics and robot's model of the environment are assigned manually. However it is possible within the same framework presented in this paper to learn the suitable links from characteristics of labels and the occupancy values.

\section{Region Segmentation}

This work focuses on semantic labeling and a high level human semantic involvement. However some additional techniques that improve the result are presented alongside. One noteworthy technique is "region segmentation". Regions in a map are those blocks of the map that have different patterns. Differences in patterns could be rooted in different structures, or even similar structures with different dominant orientations (see Fig. 8). If a map is composed of multiple regions, line sets $\mathcal{L}_{\text {ines }}$ from one region might interfere with cell decomposition of other regions, as cell decomposition is a global operation. Although theoretically the final result is still valid in such a case, yet it causes a higher decomposition and consequently degrading the abstraction level. To avoid this undesirable effect of multi regions, we exploit an unsupervised texture segmentation method based on Gabor filters proposed by J. Bigun [19]. The method proposes a Gabor filter for decomposition and feature extraction, followed by clustering of pixels according to their features. In this implementation we set the Gabor filter with 5 frequency channels and 8 orientation channels, resulting in a 40 dimension feature space. Dimensionality is reduced by Principal Component Analysis, and a k-means clustering is employed as the final step of texture segmentation. Performance of the texture segmentation method over a multi region map of a real warehouse of size $\sim 190^{m} \times 270^{m}$, fit with bounding boxes, is presented in Fig. 8 . 


\begin{tabular}{|lccc|}
\hline Region & "Total Pallets" & Correct Detection & Systematic Error \\
\hline A & 2628 & $82.6 \%$ & $11.0 \%$ \\
B & 1891 & $77.4 \%$ & $18.2 \%$ \\
C & 2172 & $92.9 \%$ & $3.2 \%$ \\
\hline
\end{tabular}

TABLE III

\begin{tabular}{|l|l|c|}
\hline Label & Functionality & Connect-ability \\
\hline co: corridor & drivable \& access & co \& ju \& of \\
\hline ju: junctions & drivable \& access & co \& ju \& of \\
\hline of: office & work place & co \& ju \& of \\
\hline un: unidentified & - & None \\
\hline
\end{tabular}

TABLE IV: Semantic labels for office environments.

\section{Results AND Discussion}

Our peoposed method improves the state of the art methodologically. The improvement is made possible by abstracting the delivery of human concept of environment to the robot, and hence avoinding a time consuming low-level training phase.

We applied our method to two different maps acquired in the warehouse of one of our industrial partners, and to one office environment map available from the Radish data set. The warehouse data was acquired in different environments and with different configurations of the sensor, resulting in different maps. In one case (Fig. 6) the range scanner is pointing at the articles stored in the pallet cells, therefore the pallet cells are mostly either occupied or unexplored. The range scanner in the other configuration is pointing to the pallets in the cell, recording the pallet footprints in the map (Fig. 7).

While all regions of the map in Fig. 8 have been tested, due to the lack of space only one region (C) is shown in Fig. 9. Table III presents a qualitative measure of the performance over the pallet cells. We skip the margin areas, as they are partially explored and the information in those regions is unreliable. Systematic error refers only to the errors caused by violation of the assumption that any place in the environment is identifiable based on its shape (i.e. any cell in the decomposition). For instance, storage cells can be empty instead of containing a pallet, or storage cells can be in unexplored areas where raycasts do not penetrate (such as when a crate is placed directly on the floor instead of on a pallet). Note that systematic error and correct detection do not add up to $100 \%$.

\section{A. Beyond Warehouses}

We showed that the method handles warehouses under differing sensor configurations. Here, we furthermore demonstrate the applicability of the concept to other kinds of environments, such as office buildings. For this purpose the method is applied to the Intel Jones Farms Campus map ${ }^{1}$. Corresponding to the change of the environment, we defined a new set of semantics (table IV). The result is shown in Fig. 10.

${ }^{1}$ This data set was obtained from the Robotics Data Set Repository (Radish) [20]. We thank Maxim Batalin for providing this data.

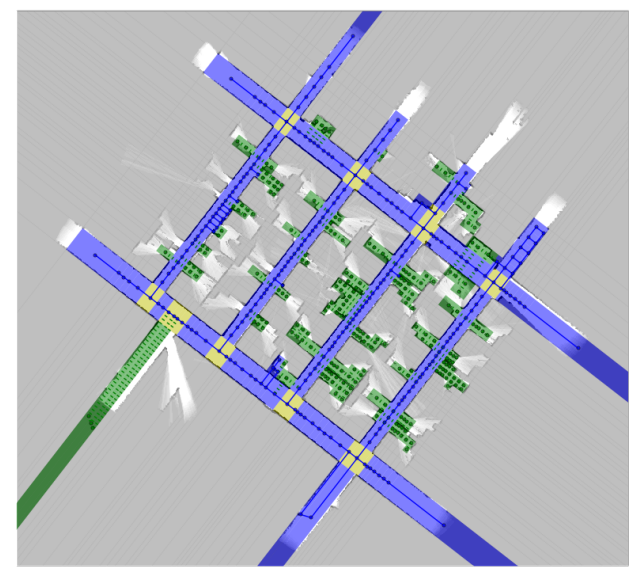

Fig. 10: Semantic map of an office-like environment obtained from the Radish data set, according to table IV.

\section{B. Discussion}

In this paper we demonstrate a method of semantic labeling of the environment, relying on a high level link between human knowledge and robot world model, using an abstract form of human semantics. The final result contains semantic annotations that are not explicitly explained to the robot, but inferred according to the linkage with human knowledge. This introduces the semantics at a higher level, which is significantly less onerous and allows the semantics to be abstracted more efficiently. We have also shown that, the method adapts with minimum effort to new kinds of environments and sensor configurations.

Outlook: The performance achieved with the current implementation corresponds to a proof of concept. Some shortcomings remain, leaving room for future improvements, some of which have been sketched in the methods section already. Some of these limitations are rooted in the shortcomings of underlying tools (e.g. k-means) and can be addressed by working on individual components. Other are more systematics, and we plan to employ combinations with other methods to overcome them.

One element of the method that significantly affects the result is the feature set, clustering, and consequently the place categorization. In [6], probabilistic relaxation is used to compensate for misclassifications. We plan to incorporate a similar scheme as well. Also we employed k-mean for clustering which does not support multi-labeling of observations in its original form. We expect multi-labeling to enable better place categorization, as some area might share similarities. For instance, corridors and junctions are both drivable and may often be very similar in the makeup of the features extracted via raycasting.

Concerning the systematic shortcomings, we plan to improve our results by augmenting a landmark map generated from vision sensor from our previous work [5]. The landmark map generated in [5] models the infrastructure of the environment (pillars) providing a more accurate spatial decomposition of the model. Here, we expect to take inspiration from 


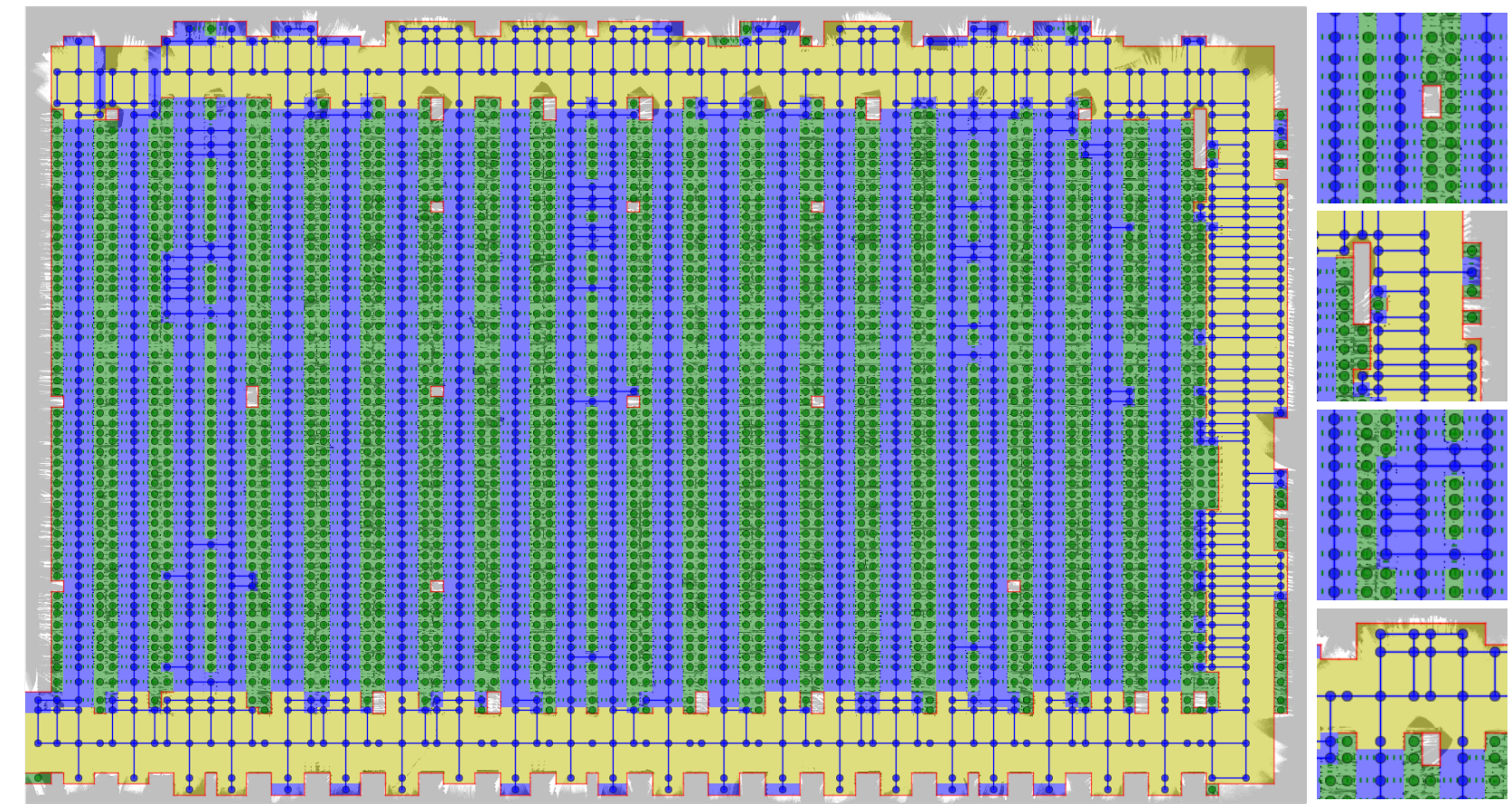

Fig. 9: Semantic map of a real warehouse, junctions in yellow, pallet cells in green and corridors in blue. Four cropped images on the right illustrate some failure cases.

research on hierarchical multi-modal methods for semantic analysis.

\section{CONCLUSION}

Robots that have an increased understanding of their environment are a key enabler for novel applications in automation, such as intelligent warehouses. In addition to increasing the reliability and robustness of operation, it can provide crucial background knowledge for adaptive learning or reasoning in novel situations. The presented work provides a reliable method of semantic interpretation of maps, supporting robots' awareness of their surroundings.

The proposed method generates a semantic map of the environment, suitable for operating autonomous robots. The problem is approached by spatial modelling of a given map, and delivering the semantics to the robot on a higher level in an abstract form. We employ a method of adaptive cell decomposition for modelling the map according to the structure of the environment. Place categorization is performed with clustering methods employing a set of simple geometric features extracted from raycasting. We present a case study of three real-world maps, one from a commonly available dataset and the others from data we collected with two different sensor setups in the warehouses of an industrial partner. The result demonstrates the simplicity and effectiveness of the method in adapting to new environments.

The key features of the proposed method are the independence from training data for semantic labels. Delivery of human semantics to the robot is done on a higher level, and directly supports semantic inference. Places are categorized in a semi-automatic unsupervised manner and are independent from the type of environments.

Improvement of the proposed method will include enhancements of place categorization and the incorporation of other modalities.

\section{REFERENCES}

[1] V. M. Monajjemi, J. Wawerla, R. Vaughan, and G. Mori, "Hri in the sky: Creating and commanding teams of uavs with a vision-mediated gestural interface," in Intelligent Robots and Systems (IROS), 2013 IEEE/RSJ International Conference on. IEEE, 2013, pp. 617-623.

[2] S. Pourmehr, V. M. Monajjemi, R. Vaughan, and G. Mori, "you two! take off!: Creating, modifying and commanding groups of robots using face engagement and indirect speech in voice commands," in Intelligent Robots and Systems (IROS), 2013 IEEE/RSJ International Conference on. IEEE, 2013, pp. 137-142.

[3] R. B. Rusu, "Semantic 3d object maps for everyday manipulation in human living environments," KI-Künstliche Intelligenz, vol. 24, no. 4, pp. 345-348, 2010.

[4] S. G. Shahbandi, B. Åstrand, and R. Philippsen, "Sensor based adaptive metric-topological cell decomposition method for semantic annotation of structured environments," in to appear in 13th International Conference on Control, Automation, Robotics \& Vision (ICARCV). [Online]. Available: http://urn.kb.se/resolve?urn=urn:nbn: se:hh:diva-26597

[5] S. G. Shahbandi and B. Åstrand, "Modeling of a large structured environment," in Advances in Autonomous Robotics Systems, ser. Lecture Notes in Computer Science, M. Mistry, A. Leonardis, M. Witkowski, and C. Melhuish, Eds. Springer International Publishing, 2014, vol. 8717, pp. 1-12. [Online]. Available: http: //urn.kb.se/resolve?urn=urn:nbn:se:hh:diva-26316

[6] O. M. Mozos, C. Stachniss, and W. Burgard, "Supervised learning of places from range data using adaboost," in Robotics and Automation, 2005. ICRA 2005. Proceedings of the 2005 IEEE International Conference on. IEEE, 2005, pp. 1730-1735.

[7] A. Rottmann, O. M. Mozos, C. Stachniss, and W. Burgard, "Semantic place classification of indoor environments with mobile robots using boosting," in proceedings of the national conference on artificial 
intelligence, vol. 20, no. 3. Menlo Park, CA; Cambridge, MA; London; AAAI Press; MIT Press; 1999, 2005, p. 1306.

[8] R. R. Vatsavai, A. Cheriyadat, and S. Gleason, "Unsupervised semantic labeling framework for identification of complex facilities in high-resolution remote sensing images," in Data Mining Workshops (ICDMW), 2010 IEEE International Conference on. IEEE, 2010, pp. 273-280.

[9] C. Farabet, C. Couprie, L. Najman, and Y. LeCun, "Learning hierarchical features for scene labeling," Pattern Analysis and Machine Intelligence, IEEE Transactions on, vol. 35, no. 8, pp. 1915-1929, 2013.

[10] S. Friedman, H. Pasula, and D. Fox, "Voronoi random fields: Extracting the topological structure of indoor environments via place labeling," in Proc. of the International Joint Conference on Artificial Intelligence (IJCAI), vol. 35, 2007.

[11] M. R. Walter, S. Hemachandra, B. Homberg, S. Tellex, and S. Teller, "A framework for learning semantic maps from grounded natural language descriptions," The International Journal of Robotics Research, vol. 33, no. 9, pp. 1167-1190, 2014.

[12] R. Capobianco, G. Gemignani, D. D. Bloisi, D. Nardi, and L. Iocchi, "Automatic extraction of structural representations of environments," in Proceedings of the 13th Intelligent Autonomous System conference, 2014.

[13] E. Bastianelli, D. D. Bloisi, R. Capobianco, F. Cossu, G. Gemignani, L. Iocchi, and D. Nardi, "On-line semantic mapping," in Advanced Robotics (ICAR), 2013 16th International Conference on, Nov 2013, pp. 1-6.

[14] A. Nüchter and J. Hertzberg, "Towards semantic maps for mobile robots," Robotics and Autonomous Systems, vol. 56, no. 11, pp. 915926, 2008.

[15] A. Ranganathan and F. Dellaert, "Semantic modeling of places using objects," in Proceedings of the 2007 Robotics: Science and Systems Conference, vol. 3, 2007, pp. 27-30.

[16] K. Joo, T.-K. Lee, S. Baek, and S.-Y. Oh, "Generating topological map from occupancy grid-map using virtual door detection," in Evolutionary Computation (CEC), 2010 IEEE Congress on. IEEE, 2010, pp. 1-6.

[17] Z. Liu and G. von Wichert, "Extracting semantic indoor maps from occupancy grids," Robotics and Autonomous Systems, vol. 62, no. 5, pp. 663-674, 2014.

[18] A. Pronobis and P. Jensfelt, "Large-scale semantic mapping and reasoning with heterogeneous modalities," in Robotics and Automation (ICRA), 2012 IEEE International Conference on. IEEE, 2012, pp. $3515-3522$.

[19] J. Bigun and J. H. du Buf, "N-folded symmetries by complex moments in gabor space and their application to unsupervised texture segmentation," Pattern Analysis and Machine Intelligence, IEEE Transactions on, vol. 16, no. 1, pp. 80-87, 1994.

[20] A. Howard and N. Roy, "The Robotics Data Set Repository (Radish)," 2003. [Online]. Available: http://radish.sourceforge.net 